\title{
PHASOR MEASUREMENT UNITS (PMUS) ALLOCATION FOR POWER SYSTEMS OBSERVABILITY
}

\author{
M.M. Shatla \\ Egyptian Electricity \\ Transmission Company \\ (EETC), Egypt
}

\author{
A.A. Abou El-Ela M.A. Abido \\ Faculty of Engineering, \\ Menoufiya University \\ Egypt
}

\author{
Ragab A. El-Sehiemy \\ Faculty of Engineering, \\ Kafr El-Sheikh University, \\ Egypt
}

\begin{abstract}
The recent development of phasor measurement units (PMUs), enhancing synchronization and high time-resolution of measurements, allows the creation of dynamic snapshots and makes network real-time observability possible. A proposed analysis of power system observability and the rules of PMUs placement with the aim of linear static state estimation (SE) of power system networks are presented in this paper. According to the Power System Analysis Toolbox (PSAT), MATLAB toolbox, several algorithms of PMUs allocations as well as their differences and relations are introduced such as a graph-theoretic procedure based on Depth First Search, Simulated Annealing Method, Minimum Spanning Tree Method, and others. These different methods are used to benchmark the real networks with different scales, topologies and voltage levels in Egypt besides the standard test systems of IEEE 14-bus and IEEE 30-bus. The obtained results indicate the effectiveness of the applied methods of optimal PMUs placement for power system observability with the aim of linear static SE as well as the further requirements for optimal PMUs placement, which are not provided by any of the different methods for future work. By comparing the studied methods, we found that, it could not be said that there is an algorithm is optimal for all cases.
\end{abstract}

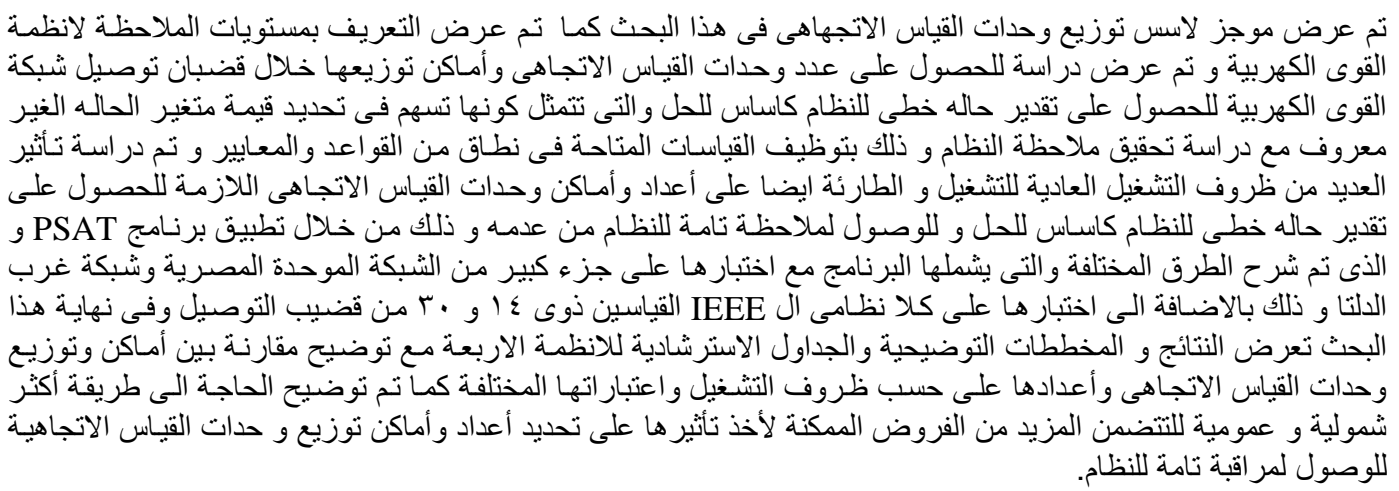

Index Terms: Phasor measurement unit (PMU), State Estimation (SE), Network Observability, Power System Analysis Toolbox (PSAT), and Synchronized Measurements 


\section{INTRODUCTION}

With the development of the power grids and the advancement of the power market, the operation of the electric power systems is becoming more and more complicated. This is mainly due to deregulated electricity market. A secure operation of the power systems requires close monitoring of the system parameters. Until recently, it was not possible to measure phase angles of the bus voltages in real-time due to the technical difficulties in synchronizing measurements from distant locations. The advent PMUs alleviate this problem by synchronizing the voltage and current waveforms at widely dispersed locations with respect to Global Positioning System (GPS) clock, which has an accuracy of less than 1 $\mu \mathrm{sec}$. This information can be used as a new way provided for the security analysis and stability control in power system operation. One of the applications of PMUs in power systems is in fault location detection [1].

The state estimation (SE) is widely used in transmission control centers. The SE provides a means of monitoring network conditions, which are not directly telemetered, and provides a valid best estimate of a consistent network model, which can be used as a starting point for real-time.

According to [2], the $\mathrm{SE}$ is the process in which one or several network parameters, whose accuracy is not certain, are obtained. However, the precise state variables cannot be obtained due to the structure complexity and the large-scale nature of the real-life power systems [3]. Recently, the PMUs are put into practice for solving power systems SE problem [4].

However, a power system is called observable only when all of its states can be uniquely determined [5]. In case of incomplete observability, the SE techniques can be applied and the rest system phasors can be predicted.

In the normal practice, it is either to install a limited number of PMUs or to install them in an incremental fashion and combine them with existing conventional measurements due to the high capital cost of PMUs. For this reason, the optimal PMUs' placement (OPP) problem can be solved in practical scenarios to make the power system topologically observable with a minimal set of PMUs .

In [6] a proposed method based on genetic algorithm has been presented to place metering devices in the network, to increase the redundancy of measurements by adding PMU to the buses in the network. A comparison of several PMU placement algorithms for SE has been presented in [7]. The PMUs placement algorithm for the SE of a power system under single measurement loss and any single-branch outage using binary integer programming has been presented in [8]. Numerous other topics are discussed in the literature relating to $\mathrm{SE}$ in power systems including robustness $[9,10]$.

The objective of the present work is to find the minimum number of PMUs to make the system observable with the aim of linear static SE of power system networks, as well as the optimal locations of these PMUs. The OPP methodologies applied include the system observablility during normal operating conditions, as well as during single branch forced outages. The PSAT is used for control and analysis of the power systems [11]. The PSAT is used to solve the OPP using different methods such as Depth First, Annealing, Direct Spanning Tree and Graph Theoretic procedure. The PSAT approach is applied to standard test systems of IEEE 14-bus and IEEE 30-bus [12], and to a real network of the 52-bus West Delta Network of 66-KV (WDN) [13], as well as the 81-bus as a part from Unified Electrical Power Network of Egypt of 220-KV (UEN). However, for benchmarking, zero-injection measurement effect is obtained for the studied test systems.

\section{LINEAR STATIC STATE ESTIMATION}

The advent of PMU technology provides an opportunity to further improve confidence in SE results. A PMU provides time-stamped measurements of active power, reactive power, frequency, current, voltage magnitude, and phase angle. The PMU 'sensor' is a GPS technology that uses conventional active and reactive power transducers as well as conventional current and voltage transformers. The PMU technology samples the waveform from 2,000 to 10,000 times per second, and then computes phasors generally around 10 to 60 times per second.

There are complementary solutions in using PMU technology with SE such as:

"Evolutionary" solution, in which improvements are achieved by adding phasor measurements to existing SE measurement set and applying 'meter placement' methods to determine most beneficial PMU locations.

"Revolutionary" solution, in which all PMU measurements are provided, this approach would require massive PMU deployment $(30 \%$ - 50\% of buses), but would allow much more frequent calculations. In fact, the "revolutionary" solution will be a natural extension of the evolutionary approach as the number of PMUs installed continues to increase.

Ref. [14] has presented different aspects of implementation of PMU technology in power system static SE. From the simulation study performed on the IEEE 14-bus test system, it has been found that PMU measurements can increase the confidence in the $\mathrm{SE}$ result. This research also has investigated the 
possibility of using a new linear SE algorithm in parallel with the existing nonlinear estimator. It has shown that this linear estimator has certain advantages.

State Estimators can generate a wealth of information, beyond that provided by system control and data acquisition (SCADA) data alone, about the present operating state of the system [15]. Phasor measurements can benefit state estimators in several ways:

First, another input measurement is available. This may improve redundancy depending upon whether the PMU is deriving its phase angle from the same current and potential transformers, which are used for measuring MW and MVAR.

More importantly, the direct measurement of a state variable (phase angle) will improve algorithmic stability and convergence. In the case where sufficient PMUs are available to provide network visibility on their own (revolutionary approach), a linear estimator can be developed which is not iterative and a high-speed estimator becomes a possibility.

The availability of PMUs in SE will no doubt enhance the ability of the estimator to detect bad data, if only by adding to redundancy. One related benefit may be to make the detection of topology errors more realistic.

Beyond the direct benefits to the SE, there are potential benefits to analytic applications, which depend upon SE results. One notable example is congestion analysis and congestion costs.

However, successful deployment of a State Estimator is not a trivial undertaking. State Estimators should be able to identify bad data, and consistently bad data should be fixed. In addition, diligent study of the State Estimator's results may lead to the identification of parameter errors. The State Estimators will also detect topology errors, perhaps indirectly, because in most cases, topology errors will produce very strange voltage solutions and consequently very strange branch flows.

By strategically locating PMUs, the effects of measurement errors can be reduced. The question is how to most effectively locate new PMUs to enhance the state estimator. It is possible to specify quantitative "condition indicators" that can be used to develop an algorithm for state estimator design. In this research, the condition indicators are used to assess PMU placement alternatives.

The static SE problem is generally formulated as a non-linear set of equations, as follows $[16,17]$ :

$$
\mathrm{z}=\mathrm{h}(\mathrm{x})+\mathrm{\epsilon}
$$

Where:

$z\left(z \in \mathbb{R}^{\mathrm{m}}\right)$ : measurement vector;

$\mathrm{x}\left(\mathrm{x} \in \mathbb{R}^{\mathrm{m}}\right)$ : state vector;

$\in\left(\in \in \mathbb{R}^{\mathrm{m}}\right)$ : measurement errors vector; and $h\left(h \in \mathbb{R}^{n} \rightarrow \mathbb{R}^{m}\right)$ : vector of the relationships between states and measurements;

Eqn. 1 is typically solved by means of a NewtonRaphson technique [16, 18].

Using devices able to provide voltage and current phasors, such as PMUs, yields a linear relationship between state variables and measurements variables, as follows:

(2)

$$
z=H(x)+\epsilon
$$

\section{Where:}

$\mathrm{H}\left(\mathrm{H}_{m} \in \mathbb{R}^{\mathrm{mxn}}\right) \mathrm{s}$ the "state" matrix of the system. Typically $m>n$, and the solution of (2) is obtained by a least mean square technique [19].

\section{POWER SYSTEM OBSERVABILITY "PMUS' PLACEMENT RULES"}

The objective of the OPP problem is the strategic choice of the minimum number of PMUs $\left(n_{P M U_{-} \min }\right)$ and the optimal allocation $A L\left(n_{P M U_{-} \min }\right)$ of the total number of PMUs $\left(n_{P M U}\right)$ in order to ensure complete observability and satisfy a preset redundancy criterion. The OPP problem can be formulated as [20]:

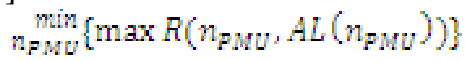

Subject to: $O_{b s}\left(n_{P M U}, A L\left(n_{P M U}\right)\right)=1$

Where $R\left(n_{P M U}, A L\left(n_{p}\right)\right)$ is the redundancy measurement index and $O_{b s}$ is the observability evaluation logical function. The optimal solution $\left(n_{P M U_{-} \text {min }}\right.$ and $A L\left(n_{P M U_{-} \text {min }}\right)$ is difficult to be obtained directly, due to the large-scale nature of the OPP combinatorial optimization problem and the dependence of system observability on the number of PMUs and the placement set. Computationally, the OPP problem is highly nonlinear, discontinuous and multi-modal, having a non-convex, and non-smooth objective function. The unobservable buses are made observable by solving the OPP considering the following conditions [20, 21]:

Condition 1: For PMU installed at a bus, the bus voltage phasors and the current phasors of all incident branches are known.

Condition 2: If one end voltage phasor and the current phasor of a branch are known, then the voltage phasor at the other end of the branch can be calculated.

Condition 3: If voltage phasors of both ends of a branch are known, then the current phasor of this branch can be directly obtained, this allows interconnecting observed zones.

Condition 4: If there is a zero-injection bus without PMU and the current phasors of the incident branches are all known except one, then the unknown current phasor can be calculated using Kirchhoff's current law( KCL), (if N-1 currents 
incident to the node are known, the last current can be computed by difference).

Condition 5: If the voltage phasor of a zeroinjection bus is unknown and the voltage phasors of all adjacent buses are known, then the voltage phasor of the zero-injection bus can be obtained through node voltage equations.

Condition 6: If the voltage phasors of a set of adjacent zero- injection buses are unknown, but the voltage phasors of all the adjacent buses to that set are known, then the voltage phasors of zeroinjection buses can be computed by node voltage equations.

\section{OPTIMAL PMUS PLACEMENT USING PSAT}

The PSAT is a Matlab toolbox for static and dynamic analysis and control of electric power systems. It includes Power Flow, continuation power flow, optimal power flow, small signal stability analysis and time domain simulation. The PSAT core is the power flow routine, which also takes care of state variable initialization. Once the power flow is solved, further static and/or dynamic analysis can be performed. The PSAT is available on-line [22]. The PSAT enables several methods for the OPP for linear static SE [23]. These methods are:

\section{A. Depth First Search (DeFS)}

An algorithm that marks all vertices in a directed graph in the order they are discovered and finished, while partitioning the graph into a forest, is called depth first search algorithm (DeFS). This method uses the Conditions 1 to 3 of Section 3, i.e. it doesn't take into account zero-injections. The first PMU is placed at the bus with the largest number of connected branches if there is more than one bus with this characteristic, one is randomly chosen. The following PMUs are placed with the same criterion, until the complete network visibility is obtained, as depicted in Fig. 1. In [24, 25], the OPP optimization problem is solved using the PSAT.

\section{B. Graph Theoretic Procedure (GTh)}

This method was originally presented in [3] and is similar to the (DeFS), except for taking into account zero-injections. Many simulations based on this method had been performed in [26].

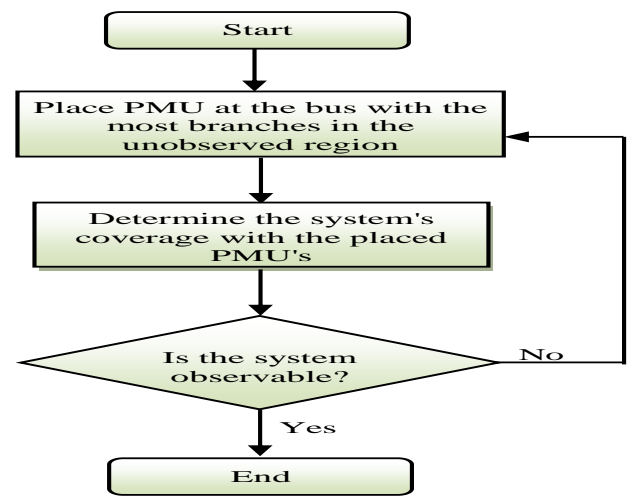

Fig. 1: Flowchart of the Graph Theoretic Procedure

\section{Simulated Annealing (SA)}

Simulated Annealing (SA) is a technique that finds a good solution to an optimization problem, by trying random variations of the current solution. A comparison of SA algorithm to others is presented in $[3,8]$. The SA flowchart is shown in Fig. 2.

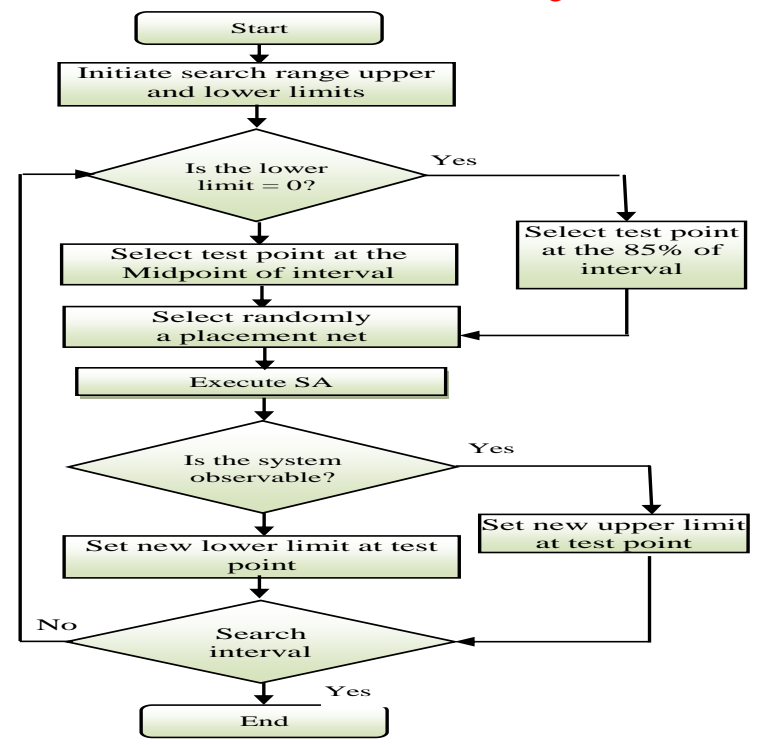

Fig. 2: Flowchart of the SA Search

D. Recursive Spanning Tree "Recursive $N$ Security Method" (Rec.SpTr)

This method is a modified depth first approach [11]. The Rec. SpTr flowchart is shown in Fig. 3. The algorithm is performed $\mathrm{N}$ times $(\mathrm{N}$ is the number of buses), using as starting bus each bus of the network. The obtained PMU sets are reprocessed as follows: one at a time, each PMU of each set is replaced at the buses connected with the node where a PMU was originally set, as depicted in Fig. 4. PMU placements which lead to a complete observability are retained. Last step is checking for condition 4. 


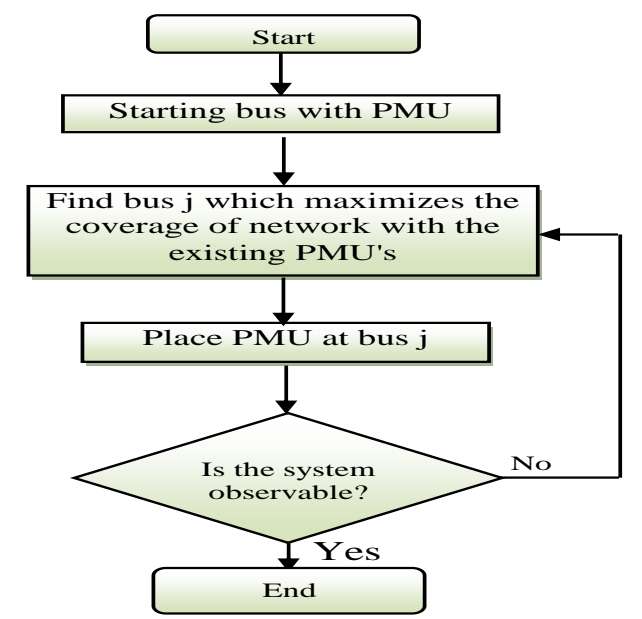

Fig. 3: Flowchart of the Recursive Spanning Tree

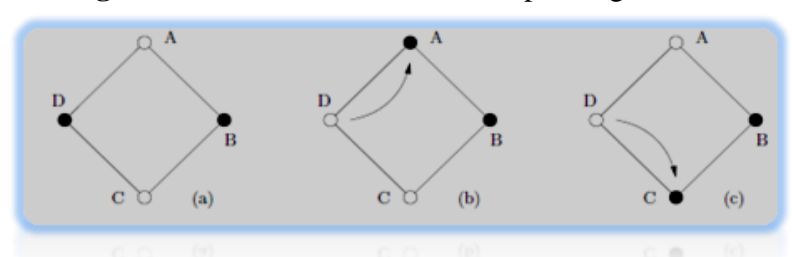

Fig. 4: Search for Alternative Placement Sets

E. Single-Shot $N$ Security Algorithm "Direct Spinning Tree" (D.SpTr)

The algorithm is based only on topological rules, and determines a single spanning tree [11]. The flowchart of the D.SpTr is shown in Fig. 5.

F. Recursive and Single-Shot Security N-1 Algorithms (Rec. N-1 SpTr \& Dir. N-1 SpTr)

Simple criteria, which yields a complete visibility in case of line outages ( $\mathrm{N}-1$ security) [11]. The flowchart of the algorithms is shown in Fig. 6.

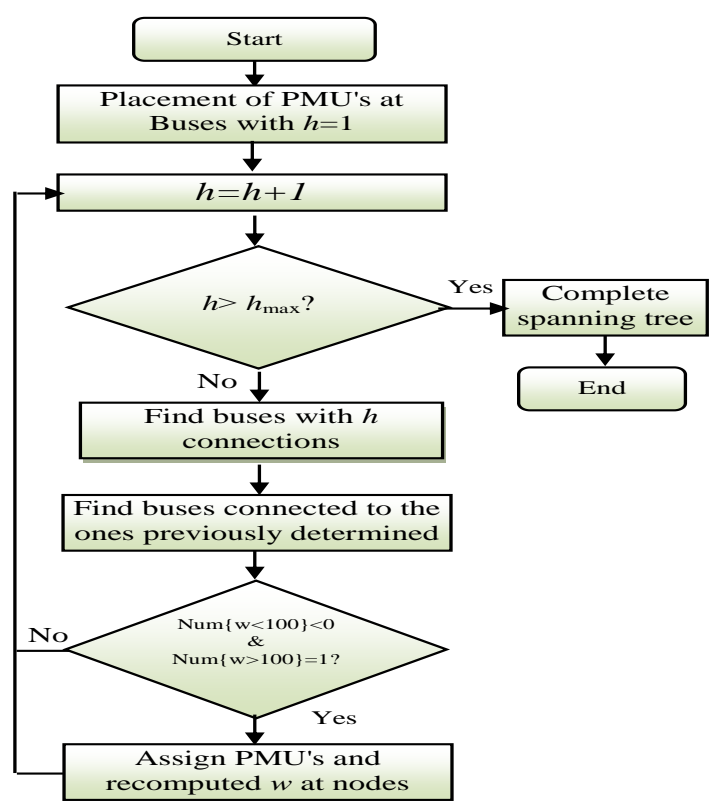

Fig. 5: Flowchart of the Single-Shot N Security Algorithm

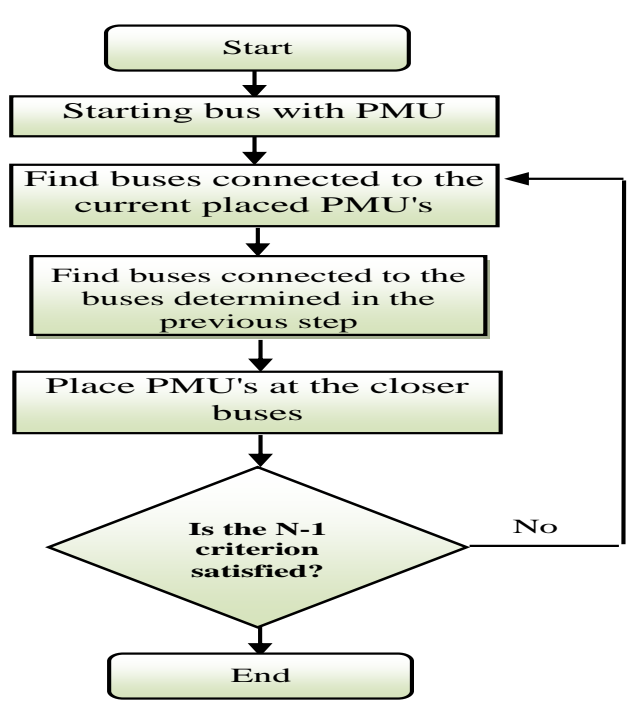

Fig. 6: Flowchart of the Recursive and Single-Shot Security (N-1) Algorithms

\section{RESULTS AND DISCUSSIONS}

The topologies of standard test systems of IEEE 14-bus and IEEE 30-bus [13] and of the real networks of the WDN [14] and the UEN are used to indicate the OPP using the different methods in the PSAT.

However, Table 1 shows the number of the branches, the redial buses, and zero-injection buses for the studied test systems.

Tables 2 and 3 show the PSAT reports for IEEE 14-Bus standard test system. It is shown that there is only one unique solution set for the different methods except with the Rec. (N-1) SpTr, which is giving more than one optional solution set to the system operator, as indicated in Table 3.

Table 4 shows the OPP results obtained from the different methods of the PSAT applied on the test systems for the linear static SE.

TABLE 1 Number of Branches, Redial and Zero-Injection Buses for the Studied Test Systems

\begin{tabular}{|l|ll|l|}
\hline Studied System & $\begin{array}{l}\text { No. } \\
\text { branches }\end{array}$ & $\begin{array}{r}\text { of No. of } \\
\text { radial Buses }\end{array}$ & $\begin{array}{r}\text { of } \\
\text { injection buses }\end{array}$ \\
\hline IEEE 14-BUS & 20 & 1 & 1 \\
\hline IEEE 30-BUS & 41 & 3 & 6 \\
\hline WDN 52-BUS & 55 & 17 & 0 \\
\hline UEN 81-BUS & 79 & 13 & 0 \\
\hline
\end{tabular}


TABLE 2 PMUs' Placements for the 14-Bus Test System using Different PSAT Methods Giving one Solution Set

\begin{tabular}{|l|l|l|l|l|l|l|}
\hline \multicolumn{7}{|c|}{ *PMUs' PLACEMENT Using: } \\
\hline Bus & $\begin{array}{l}\text { DeF } \\
\text { S }\end{array}$ & GTh & SA & $\begin{array}{l}\text { D.Sp } \\
\text { Tr }\end{array}$ & $\begin{array}{l}\text { Rec.Sp } \\
\text { Tr }\end{array}$ & $\begin{array}{l}\text { Dir. (N- } \\
\text { 1) SpTr }\end{array}$ \\
\hline $\mathbf{1}$ & $\mathbf{1}$ & $\mathbf{1}$ & $\mathbf{1}$ & 0 & 0 & 0 \\
\hline $\mathbf{2}$ & 0 & 0 & 0 & $\mathbf{1}$ & $\mathbf{1}$ & $\mathbf{1}$ \\
\hline $\mathbf{3}$ & 0 & 0 & 0 & 0 & 0 & $\mathbf{1}$ \\
\hline $\mathbf{4}$ & $\mathbf{1}$ & $\mathbf{1}$ & $\mathbf{1}$ & 0 & 0 & 0 \\
\hline $\mathbf{5}$ & 0 & 0 & 0 & 0 & 0 & $\mathbf{1}$ \\
\hline $\mathbf{6}$ & $\mathbf{1}$ & $\mathbf{1}$ & 0 & 0 & $\mathbf{1}$ & $\mathbf{1}$ \\
\hline $\mathbf{7}$ & 0 & 0 & 0 & $\mathbf{1}$ & 0 & $\mathbf{1}$ \\
\hline $\mathbf{8}$ & $\mathbf{1}$ & 0 & 0 & 0 & 0 & 0 \\
\hline $\mathbf{9}$ & 0 & 0 & 0 & 0 & $\mathbf{1}$ & 0 \\
\hline $\mathbf{1 0}$ & $\mathbf{1}$ & $\mathbf{1}$ & $\mathbf{1}$ & 0 & 0 & $\mathbf{1}$ \\
\hline $\mathbf{1 1}$ & 0 & 0 & 0 & $\mathbf{1}$ & 0 & 0 \\
\hline $\mathbf{1 2}$ & 0 & 0 & 0 & 0 & 0 & 0 \\
\hline $\mathbf{1 3}$ & 0 & 0 & $\mathbf{1}$ & $\mathbf{1}$ & 0 & $\mathbf{1}$ \\
\hline $\mathbf{1 4}$ & $\mathbf{1}$ & $\mathbf{1}$ & 0 & 0 & 0 & $\mathbf{1}$ \\
\hline
\end{tabular}

* Note: 1 donates that there is a PMU at the Bus while 0 means no PMU

TABLE 3 PMUs' Placements for the 14-Bus Test System Using Rec. (N-1) Spanning Tree For The Solution Sets PMU PLACEMENT

\begin{tabular}{|l|l|l|l|l|l|l|l|l|l|l|}
\hline Bus & Set1 & Set2 & Set3 & Set4 & Set5 & Set6 & Set7 & Set8 & Set9 & Set10 \\
\hline
\end{tabular}

\begin{tabular}{|l|l|l|l|l|l|l|l|l|l|l|}
\hline 1 & 0 & $\mathbf{1}$ & $\mathbf{1}$ & 0 & 0 & $\mathbf{1}$ & 0 & 0 & 0 & $\mathbf{1}$ \\
\hline 2 & $\mathbf{1}$ & 0 & $\mathbf{1}$ & $\mathbf{1}$ & $\mathbf{1}$ & $\mathbf{1}$ & $\mathbf{1}$ & $\mathbf{1}$ & $\mathbf{1}$ & $\mathbf{1}$ \\
\hline 3 & 0 & $\mathbf{1}$ & 0 & $\mathbf{1}$ & $\mathbf{1}$ & 0 & $\mathbf{1}$ & $\mathbf{1}$ & $\mathbf{1}$ & 0 \\
\hline 4 & 0 & 0 & $\mathbf{1}$ & 0 & 0 & $\mathbf{1}$ & 0 & 0 & 0 & $\mathbf{1}$ \\
\hline 5 & $\mathbf{1}$ & $\mathbf{1}$ & 0 & $\mathbf{1}$ & $\mathbf{1}$ & 0 & $\mathbf{1}$ & $\mathbf{1}$ & $\mathbf{1}$ & 0 \\
\hline 6 & $\mathbf{1}$ & 0 & $\mathbf{1}$ & 0 & 0 & $\mathbf{1}$ & 0 & 0 & $\mathbf{1}$ & $\mathbf{1}$ \\
\hline 7 & $\mathbf{1}$ & $\mathbf{1}$ & $\mathbf{1}$ & $\mathbf{1}$ & $\mathbf{1}$ & $\mathbf{1}$ & $\mathbf{1}$ & $\mathbf{1}$ & $\mathbf{1}$ & $\mathbf{1}$ \\
\hline 8 & 0 & 0 & 0 & 0 & 0 & 0 & 0 & 0 & 0 & 0 \\
\hline 9 & $\mathbf{1}$ & $\mathbf{1}$ & 0 & $\mathbf{1}$ & $\mathbf{1}$ & 0 & $\mathbf{1}$ & $\mathbf{1}$ & $\mathbf{1}$ & 0 \\
\hline 10 & $\mathbf{1}$ & 0 & $\mathbf{1}$ & 0 & 0 & $\mathbf{1}$ & 0 & 0 & 0 & $\mathbf{1}$ \\
\hline 11 & 0 & $\mathbf{1}$ & 0 & $\mathbf{1}$ & $\mathbf{1}$ & 0 & $\mathbf{1}$ & $\mathbf{1}$ & $\mathbf{1}$ & 0 \\
\hline 12 & 0 & $\mathbf{1}$ & 0 & $\mathbf{1}$ & $\mathbf{1}$ & 0 & $\mathbf{1}$ & $\mathbf{1}$ & 0 & $\mathbf{1}$ \\
\hline 13 & $\mathbf{1}$ & $\mathbf{1}$ & $\mathbf{1}$ & $\mathbf{1}$ & $\mathbf{1}$ & $\mathbf{1}$ & $\mathbf{1}$ & $\mathbf{0}$ & $\mathbf{1}$ & 0 \\
\hline 14 & $\mathbf{1}$ & 0 & $\mathbf{1}$ & 0 & 0 & $\mathbf{1}$ & 0 & $\mathbf{1}$ & 0 & $\mathbf{1}$ \\
\hline
\end{tabular}

* Note: 1 donates that there is a PMU at the Bus while 0 means no PMU

TABLE 4 The PSAT Results for the OPP for Linear SE

\begin{tabular}{|c|c|c|c|c|}
\hline \multirow[b]{2}{*}{ Method } & \multicolumn{4}{|c|}{ PSAT OPP result for: } \\
\hline & $\begin{array}{l}\text { 14- } \\
\text { Bus }\end{array}$ & 30-Bus & WDN & UEN \\
\hline Depth First (DeFS) & 6 & 12 & 18 & 34 \\
\hline $\begin{array}{lll}\text { Graph } & \text { Theoretic } & \text { Procedure } \\
\text { (GTh) } & & \\
\end{array}$ & 5 & 11 & 18 & 33 \\
\hline Annealing Method (SA) & 4 & $7 *$ & $18^{*}$ & $34 *$ \\
\hline $\begin{array}{llll}\begin{array}{l}\text { Rec. } \\
\text { SpTr) }\end{array} & \text { Spanning } & \text { Tree } & \text { (Rec. } \\
\end{array}$ & 3 & 7 & 17 & 26 \\
\hline 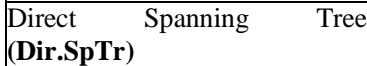 & 4 & 10 & 18 & 27 \\
\hline Rec. (N-1) Spanning Tree & 8 & 16 & 24 & 41 \\
\hline $\begin{array}{l}\begin{array}{l}\text { Direct (N-1) Spanning } \\
\text { (Dir. N-1 SpTr) }\end{array} \\
\end{array}$ & & 17 & 29 & 44 \\
\hline
\end{tabular}

Note: * donates that there is no convergence for complete depth of observability

The Single-line diagrams (SLDs) for the four studied systems are shown in Figs. 7-10 with the PMUs distributions as per the PSAT outputs for the OPP using the SA.
However, from Tables 2 to 4, the OPP results from the PSAT on studied test systems the indicate that:

i. $\quad$ No specified technique in the PSAT could be considered as absolute optimal technique for all cases؛

ii. The Depth First Search gives the highest PMUs number because it does not consider the zero injections and further its solution is not optimal!

iii. In case of not considering the zero-injections, the $\mathrm{SA}$ is giving the minimum numbers of PMUs, but it suffers from inconvenience regarding complete observability in large systems;

iv. Less number of PMUs will be required if the zero-injections buses are considered!

v. By the Rec. Spanning Tree algorithm, lower number of PMUs is obtained rather than Dir. Spanning Tree and further Rec. SpTr is providing optional solution sets for the OPP؛

vi. In case of any single branch forced outage, higher number of the PMUs is required and hence the cost will be increased!

vii. In case of studying single branch outage, the Rec. (N-1) Spanning Tree is better than the Dir. (N-1) Spanning Tree!

viii. However, the case of a single PMU forced outage is not considered by any of the methods of the PSAT!

ix. From all the applied algorithms no technique enables to select the OPP according to the strategically importance of the buses such as a bus connected to a heavily loaded or economically important area, or a bus that is a possible future expansion point; and

x. Further, the applied methods are not flexible for the selecting of the observability depth.

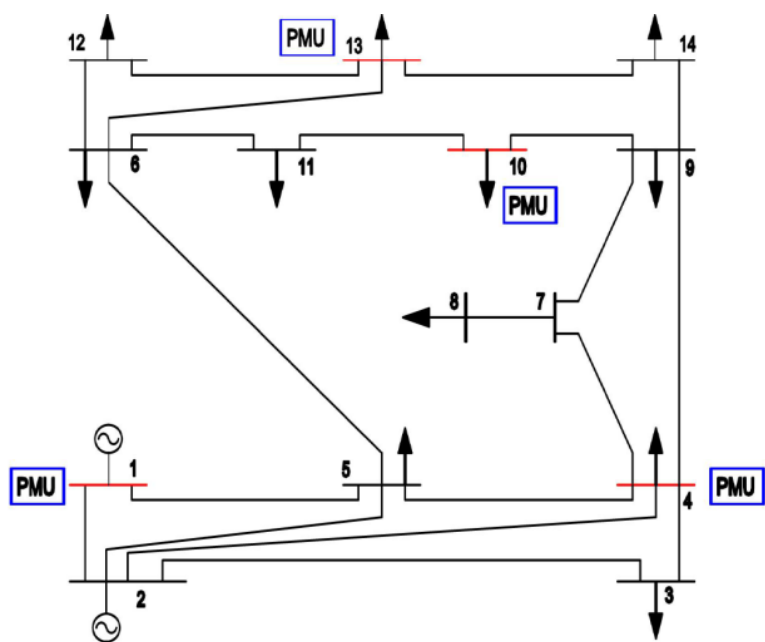

Fig.7: SLD of the IEEE 14-Bus Standard Test System with PMUs Distributions 


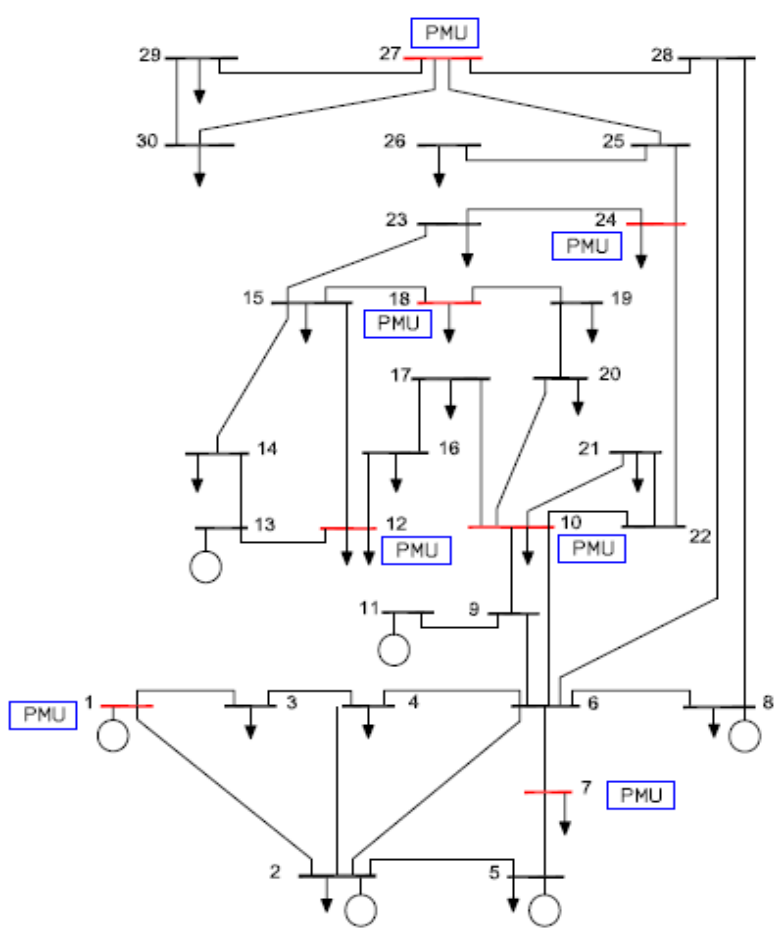

Fig. 3.8: SLD of the IEEE 30-Bus Standard Test System with PMUs Distributions using SA

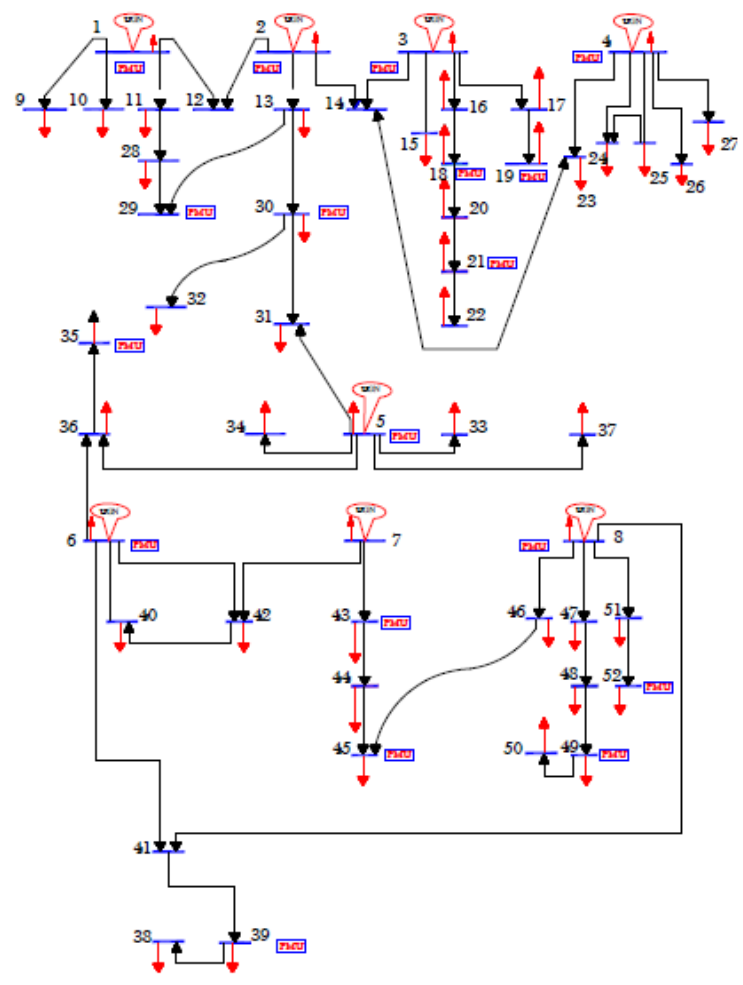

Fig. 3.9: SLD of the 52-Bus WDN with PMUs Distributions using SA

\section{CONCLUSION}

In this paper, the problem of determining the minimum number of the PMUs and their placement to ensure that a power system is a topologically observable with the aim of linear static SE has been presented. Single branch outages as well as normal operating condition have been efficiently studied. The PSAT has been applied to test different algorithms to solve the OPP for IEEE standard 14bus, 30-bus test systems and for a real networks with different voltages rating in Egypt which are the WDN and the UEN. The OPP results have been discussed to demonstrate the effectiveness of the different applied methods. Several factors such as the number and location of zero injection buses, the number and location of installed PMUs, and the system topology have been accomplished the OPP. Fewer number of PMUs may be needed if there are zero injection buses in the system. The results of this paper can be extended to investigate the benefits of adding a small number of PMUs at strategic locations to improve the monitoring and control capability in the system. The obtained information for PMUs can be used as a new way provided for the security analysis and stability control in power system operation. The optimal PMU placement decreases the number of PMUs, which redounds cost declining, and obtains better power network operation and monitoring.

\section{REFERENCES}

[1] A. H. Al-Mohammed, M. M. Mansour, and M. A. Abido, "Application of Phasor Measurement Units (PMUs) for Fault Location in SEC-EOA Interconnected Network," Proceedings of the IEEE Energycon, Manama, Bahrain, December 18-22, pp. 435-439, 2010

[2] Xu, B. Abur, A. "Observability Analysis and Measurement Placement for Systems with PMUs," Power Systems Conference and Exposition, 2004. IEEE PES on Oct. 2002 vol 2, page(s): 943-946.2004.

[3] T. L. Baldwin L. Mili M. B. Boisen, Jr. R. Adapa "Power System Observability with Minimal Phasor Measurement Placement" IEEE Transactions on Power Systems, Vol. 8, No. 2, May 1993.

[4] Abhinav Sadu, Rajesh Kumar and Rajesh G. Kavasseri. "Optimal Placement of Phasor Measurement Units using Particle Swarm Optimization”, 2009 World Congress on Nature \& Biologically Inspired Computing (NaBIC 2009). 
[5] A. Abur and A. G. Exposito, "Power System State Estimation: Theory and Implementation," New York: Mercel Dekker, 2004

[6] Goser J., Rolim J.G., Simoes Costa A.J.A. "Meter Placement for Power System State Estimation: An Approach Based on Genetic Algorithms and Topological Observability Analysis," Proc. of the ISAP'2001 Conference. Budapest, Hungary, pp. P. 15-16, 2001

[7] Xu Dongjie, He Renmu, Wang Peng, and $\mathrm{Xu}$ Tao, "Comparison of Several PMU Placement Algorithms for State Estimation," The Institution of Electrical Engineers (C2004. Printed and published by the IEE, Michael Faraday House, Six Hills Way, Stevenage, SG1 2AY, pp. 32-35, 2004

[8] Chawasak Rakpenthai, Suttichai Premrudeepreechacharn, Sermsak Uatrongjit, and Neville R. Watson, “An Optimal PMU Placement Method Against Measurement Loss and Branch Outage," IEEE Transactions on Power Delivery, Vol. 22, No. 1, pp. 101-107, January 2007

[9] S. Pajic and K. A. Clements, "Power system state estimation via globally convergent methods," IEEE Transactions on Power Systems, vol. 20, pp. 1683-1689, 2005

[10] P. Ristanovic, "State estimation based realtime markets - challenges and practical solutions," pp. 2849-2850 Vol. 3. (2005)

[11] Federico Milano, Power System Analysis Toolbox (PSAT), Documentation for PSAT version 1.3.4, July 14, 2005

[12] R. Christie, Power System Test Archive, Aug. $1999 . \quad$ [Online].Available: http://www.ee.washington.edu/research/pstca

[13] A.A. Abou El-Ela, S.M. Allam and M.M. Shatla "Maximal optimal benefits of distributed generation using genetic algorithms," Electric Power Systems Research ,pp. 869-877, 80 (2010)

[14] Zivanovic, R, Cairns, C. "Implementation of PMU Technology in State Estimation: An Overview," IEEE PES 0-7803-3019-6/96. (2006)

[15] Allemong, J. "State estimation fundamentals for successful deployment," Power Engineering Society General Meeting. IEEE June 12-16, Page(s): 2203 - 2204. (2005).

[16] Schweppe, F., Wildes, J. and Rom, D., "Power System Static-State Estimation," Part I, II, III, IEEE Transactions on Power Apparatus and Systems 89(1), 120-135. (1970)

[17] Clements, K., Krumpholz, G. and Davis, P., "Power System State Estimation Residual Analysis: An Algorithm Using Network Topology," IEEE Transactions on Power Apparatus and Systems 100(4), 1779-1787. (1981)
[18] Allemong, J., Radu, L. and Sasson, A., “A Fast and Reliable Estimation Algorithm for AEP's New Control Center," IEEE Transactions on Power Apparatus and Systems 101(4), 933-944.(1982)

[19] Thorp, J., Phadke, A. and Karimi, K., "Real Time Voltage Phasor Measurement for Static State Estimation," IEEE Transactions on Power Apparatus and Systems 104(11), 3908-3103.(1985)

[20] J. Peng, Y. Sun, and H. F. Wang, "Optimal PMU placement for full network observability using Tabu search algorithm," International Journal of Electrical Power \& Energy Systems, vol. 28, no. 4, pp. 223-231, May 2006

[21] B. Mohammadi-Ivatloo, “Optimal placement of PMUs for power system observability using topology based formulated algorithm," Journal of Applied Sciences, Vol. 9, No. 13, pp. 2463-2468, 2009

[22] PSAT, Available [Online]: http://thunderbox.uwaterloo.ca/fmilano

[23] Morteza Farsadil, Hamid Golahmadi and Hadi Shojaei, "PMU Allocation in Power System with Different Algorithms," IEEE Transactions on Power Systems, pp. 396-400, May, 2009

[24] Morteza Farsadil, Hamid Golahmadi and Hadi Shojaei, "PMU Allocation in Power System with Different Algorithms," IEEE Transactions on Power Systems, pp. 396-400, May, 2009

[25] G. Venugopal, R. Veilumuthu, and P. Avila Theresa, "Optimal PMU placement and observability of power system using PSAT," Joint Journal Conf. on Engineering and Technology, pp.67-71, 2010

[26] Y. Yang, H. Shu, and L. Yue, "Engineering practical method for PMU placement of 2010 Yunnan power grid in China," Int. Conf. on Sustainable Power Generation and Supply, pp. 1-6, 2009 


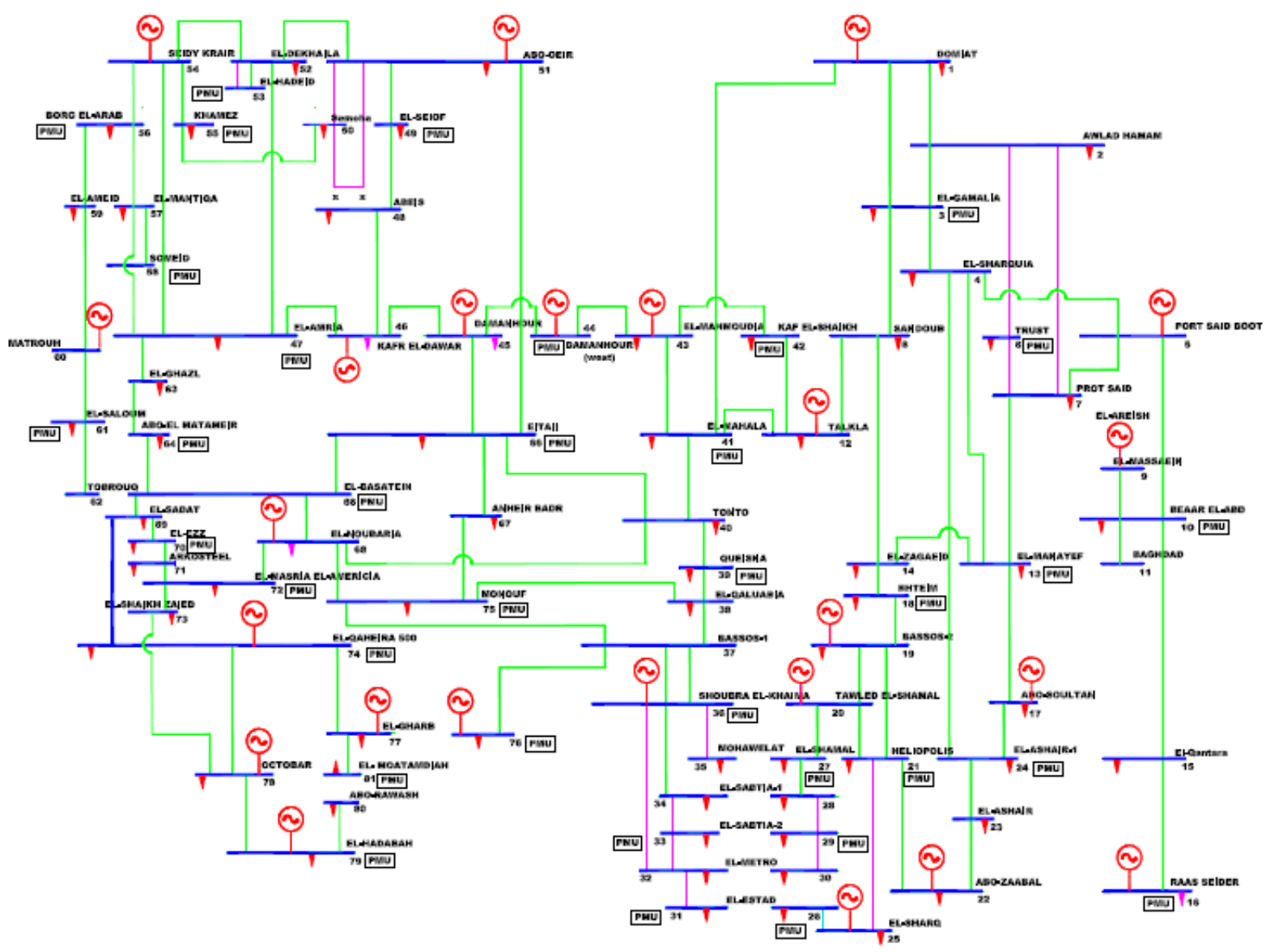

Fig. 10: SLD of the 81-Bus UEN with PMUs Distributions using SA 